\title{
Incidence and Risk Factors for Implant Failure in Spinal Metastasis Surgery
}

\author{
Yu Chung Wong ${ }^{1}$, Wai Wang Jacky Chau ${ }^{2}$, Kin On Kwok ${ }^{1}$, Sheung Wai Law ${ }^{1}$ \\ ${ }^{1}$ Department of Orthopedics and Traumatology, Prince of Wales Hospital, Hong Kong SAR, China \\ ${ }^{2}$ Department of Orthopedics and Traumatology, The Chinese University of Hong Kong, Hong Kong SAR, China
}

\section{Study Design: Retrospective study.}

Purpose: To investigate the incidence of symptomatic and asymptomatic implant failure in spinal metastasis surgery and identify potential risk factors.

Overview of Literature: Surgical stabilization with instrumentation is an established method for the treatment of spinal metastasis. However, very few studies have investigated the incidence and risk factors for implant failure after spinal instrumentation surgery for the treatment of spinal metastasis.

Methods: This study recruited 88 patients who received surgical stabilization with instrumentation for the treatment of spinal metastasis. Their medical records and postoperative X-rays were reviewed for evidence of implant failure. Statistical analysis with logistic regression was performed to assess nine potential risk factors for the development of implant failure, including patient's age at operation, gender, survival, primary tumor, spinal level involved, construct length, decompression levels, fusion material utilization, and radiotherapy application either before or after surgery, to identify potential contributing risk factors.

Results: Implant failure was identified in nine out of 88 cases (10.2\%) with two cases requiring implant removal: one case included a progressive kyphosis that resulted in nonhealing sore and the other involved a deep-seated wound infection that spread to the implants. Another case required wound debridement due to superficial wound infection. The remaining six cases were asymptomatic, despite postoperative $\mathrm{X}$-rays demonstrating evidence of implant failure. No patient required implant revision. Logistic regression analysis demonstrated that patients who received radiotherapy either before or after surgery were less likely to develop implant failure.

Conclusions: The development of radiological implant failure following surgical treatment of spinal metastasis is common. However, symptomatic implant failure leading to revision surgery is uncommon. Our findings suggest that radiotherapy, either before or after spinal surgery, is not associated with the development of implant failure.

Keywords: Neoplasm metastasis; Spine; Radiotherapy; Reoperation

\section{Introduction}

The most common site for skeletal metastasis is the spinal column [1], and treatment options include analgesics, radiotherapy, chemotherapy, hormonal therapy, and surgery [2]. Decompression and surgical stabilization with in-

Received Jan 29, 2020; Revised May 8, 2020; Accepted Jun 3, 2020

Corresponding author: Yu Chung Wong

Department of Orthopedics and Traumatology, Prince of Wales Hospital, 30-32 Ngan Shing Street, Shatin, NT, Hong Kong

Tel: +852-35052211, Fax: +852-26377889, E-mail: ycwong@ort.cuhk.edu.hk 
strumentation is an established method for the treatment of spinal metastasis [3]. Different surgical strategies have been proposed, including decompression with stabilization, with or without an attempt to remove or reconstruct the whole tumor bulk (en bloc resection) [4]. The advent of stereotactic radiosurgery makes the delivery of highdose radiation to a confined volume of tumor tissue possible [5], which led to the introduction of "separation surgery," aiming to separate the spinal cord from the tumor tissue with stability provided solely by the posterior instrumentation, while the tumor bulk is controlled by high-dose radiation rather than surgical removal [6].

In instrumented fusion for the treatment of nonmetastatic conditions of the spine, fusion materials in the form of artificial or autologous bone graft are frequently used to achieve long-term fusion to prevent the development of implant failure, deformity, or pain [7]. However, in the surgical treatment of spinal metastasis, the use of fusion materials is optional, as patients with spinal metastasis often have a limited life expectancy and implant failure may not develop within their remaining lifespan $[8,9]$.

Studies in the literature regarding implant failure after surgical stabilization of spinal metastasis are limited [1015]; therefore, this study aims to investigate the incidence of implant failure after surgical stabilization of spinal metastasis with spinal instrumentation and analyze the potential risk factors in the development of implant failure.

\section{Materials and Methods}

\section{Participants}

This study recruited 88 patients who received surgical stabilization with instrumentation for spinal metastasis from malignant tumor at the orthopedic unit of the Prince of Wales Hospital, Hong Kong from 2007 to 2017. The study was approved by the ethics review board of the Joint New Territories East Cluster/Chinese University of Hong Kong Ethics Committee (reference number: 2019.620). Only cases with at least one postoperative X-ray were included in the study. The spinal levels affected by metastasis ranged from the $\mathrm{C} 2$ level down to L5. In some patients, there were multiple levels of metastasis across or within the cervical, thoracic, or lumbar spine, requiring longsegment stabilization. Table 1 presents the levels of spinal involvement and the approaches used.

In addition to the patient's demographic data, survival
Table 1. Levels of spinal involvement and surgical approaches

\begin{tabular}{lcccc} 
Spinal levels & $\begin{array}{c}\text { Anterior } \\
\text { approach }\end{array}$ & $\begin{array}{c}\text { Posterior } \\
\text { approach } \\
\text { approach with } \\
\text { additional } \\
\text { anterior } \\
\text { support }\end{array}$ & $\begin{array}{c}\text { Total } \\
\text { no. of } \\
\text { patients }\end{array}$ \\
\hline Cervical & 8 & 9 & 0 & 17 \\
\hline Cervical+thoracic & 0 & 1 & 0 & 1 \\
\hline Thoracic & 5 & 34 & 7 & 46 \\
\hline Thoracic+lumbar & 0 & 5 & 0 & 5 \\
\hline Lumbar & 2 & 16 & 1 & 19 \\
\hline
\end{tabular}

Table 2. Potential contributing factors for the development of implant failure

\begin{tabular}{lc} 
Variable & \multicolumn{1}{c}{ Value } \\
Construct length (spinal levels) & 5.29 (range, 3-15) \\
\hline Decompression level & 1.14 (range, 0-3) \\
Use of fusion materials & \\
\hline Yes & 35 \\
\hline No & 53 \\
\hline Radiotherapy before or after operation & \\
\hline Yes & 84 \\
\hline No & 4 \\
\hline
\end{tabular}

Values are presented as average (range) or number.

time, primary tumor type, spinal involvement levels, and fusion material use were also identified. The following potential contributing factors to the development of implant failure after spinal metastasis surgery were examined (Table 2).

\section{1) Construction length}

It is defined as the number of spinal levels the implants spanned across the whole construct. For example, a patient with cervical spinal metastasis who had a corpectomy at C6 with reconstruction of the C6 vertebral body, with a cage and anterior cervical plate stabilization and screw fixation at the $\mathrm{C} 5$ and $\mathrm{C} 7$ levels, has a construct length of three spinal levels. A patient with spinal metastasis at the T7 level with decompression and posterior spinal fusion with pedicle screws and rod fixation at T5, T6, $\mathrm{T} 8$, and $\mathrm{T} 9$ levels is regarded as having a construct length of five spinal levels.

\section{2) Decompression}

A patient who received corpectomy at $\mathrm{C} 6$ with plate fixa- 
tion that spanned across C5 and C7 is regarded as having a single-level decompression. A patient with metastasis at the T7 and T8 levels, with laminectomy across T7 and T8 and posterior spinal fusion from T5 to T10, is regarded as having a two-level decompression. Most of the patients who received surgery had decompression procedures in addition to stabilization. However, some patients received prophylactic stabilization due to spinal instability before they developed cord compression. In these patients, no decompression procedure was performed.

3) Radiotherapy (either before or after the spinal operation) All of the patients included in the study had postoperative radiographs taken. Some of them also had cross-sectional imaging, including computed tomography (CT) of the thorax and abdomen or a positron emission tomography scan for systemic staging or follow-up of the tumor. Imaging studies of all patients were reviewed for evidence of implant failure defined as follows: (1) displacement of implant position, such as cage subsidence or screws pulled out; (2) change in spinal alignment, with an increase in sagittal angulation of the construct by more than $5^{\circ}[10]$; (3) signs of implant loosening, such as development of halos around the screws; and (4) implant breakage.

For those patients who developed radiographic features of implant failure, their medical records were also reviewed to determine whether they had developed any symptoms and whether revision surgery was required.

\section{Statistical analysis}

Nine potential risk factors for the development of implant failure after spinal metastasis surgery were analyzed: age at operation, survival time after surgery, gender, primary tumor, level of spinal metastasis, construct length, number of spinal level decompression, use of fusion material, and use of radiotherapy either before or after the operation.

The "primary tumor" variable included 19 different types, with some tumor types only appearing in a single patient. To facilitate statistical analysis, the tumor types were stratified into three categories: slow growth, moderate growth, and rapid growth (Table 3). The stratification was based on the scoring systems of the Tomita Score and the Katagiri Score [16,17]. For example, nasopharyngeal cancer is a common tumor in Hong Kong and was stratified as a slow-growth tumor in accordance with the recommendation made by a locally published paper on
Table 3. Stratification of primary tumors

\begin{tabular}{|lc|}
\hline Tumor stratification and subtypes & No. of patients \\
\hline Slow growth & 17 \\
\hline Breast & 9 \\
\hline Prostate & 2 \\
\hline Thyroid & 8 \\
\hline Myeloma & 1 \\
\hline Lymphoma & 4 \\
\hline Plasmacytoma & 2 \\
\hline Nasopharyngeal carcinoma & 43 \\
\hline Subtotal & \\
\hline Moderate growth & 4 \\
\hline Kidney & 1 \\
\hline Uterus & 3 \\
\hline Colorectal & 8 \\
\hline Subtotal & 25 \\
\hline Rapid growth & 1 \\
\hline Lung & 1 \\
\hline Stomach & 4 \\
\hline Bladder & 2 \\
\hline Sarcoma & 1 \\
\hline Uterine cervix & \\
\hline Unknown tumor & \\
\hline Subtotal & \\
\hline
\end{tabular}

Table 4. Stratification of levels of spinal metastasis

\begin{tabular}{lc} 
Variable & No. of patients \\
\hline Semi-rigid spine (T3-10) & 35 \\
Mobile spine (C3-6, L2-4) & 22 \\
Junctional region (CO-2, C7-T2, T11-L1, L5-S1) & 31 \\
\hline
\end{tabular}

the predictive value of the modified Tokuhashi score in a Southern Chinese population [18].

The level of spinal metastasis was also stratified into three categories in accordance with the Spinal Instability Neoplastic Score: semirigid spine, mobile spine, and junctional region (Table 4) [19]. In patients with multiple levels of spinal involvement, the spinal level subjected to the highest stress was chosen for analysis. For example, a patient with simultaneous metastatic involvement of T9 and T12 was regarded as having metastasis in the junctional region rather than a semirigid spine. 
Statistical analysis with "logistic regression" was made using IBM SPSS Statistics software ver. 25.0 (IBM Corp., Armonk, NY, USA), with the development of implant failure as the dependent variable and the nine potential risk factors as independent variables. The strength of relationship was decided by odds ratio. A two-sided $p$-value of $\leq 0.05$ was considered statistically significant.

Seven patients remained alive at the time of data retrieval on September 13, 2019. In the statistical analysis of survival time as an independent variable, these seven patients' survival periods were counted from the time of their index operation to September 13, 2019.

\section{Results}

There were 45 male and 43 female patients. The average age of the patients at the time of operation was 57.3 years old. Seven of 88 patients survived at the time of data retrieval on September 13, 2019. The median survival time for those patients who had passed away at the time of data retrieval was 17.2 months (range, 1-115.8 months) (Table 5 ). Four patients' primary tumors were not identified, despite attempts at primary tumor workup and histological assessment of surgical specimens obtained during spinal surgery. The remaining 84 patients had one of 19 different primary tumors (Table 3 ).

An anterior surgical approach was performed in 19 patients, with eight cases involving the subaxial cervical spine. A standard Smith-Robinson method was used in the anterior approach to the cervical spine. After removal of the tumor, a metal mesh cage was inserted to reconstruct the vertebral body, and an anterior cervical plate was added, spanning one spinal level above and one

Table 5. Patients' demographics

\begin{tabular}{|c|c|}
\hline Variable & Value \\
\hline Total no. of patients & 88 \\
\hline Mean age at operation (yr) & $57.3 \pm 10.6$ \\
\hline \multicolumn{2}{|l|}{ Gender } \\
\hline Male & 45 \\
\hline Female & 43 \\
\hline Deceased within the study period & $81(93.1)$ \\
\hline Median survival for the deceased cases (mo) & 17.2 (range, 1-115.8) \\
\hline
\end{tabular}

Values are presented as number, mean \pm standard deviation, number (\%), or median (range). below. Two cases of T1 tumor were also treated using the Smith-Robinson approach, with one case requiring manubriotomy for exposure. There were three cases of midto lower thoracic tumor (T5, T6, and T10 levels), treated using the anterior transthoracic approach. An anterior retroperitoneal approach was used for two cases of tumor involvement of the lumbar spine at L4 and L5, respectively. In the seven cases of thoracicolumbar spinal tumor with anterior exposure, a metal mesh cage was used to reconstruct the vertebra without additional anterior plating or posterior stabilization.

A posterior-only stabilization was used in 65 cases. There were three cases of occipital-cervical fusion with occipital plate and cervical spine pedicle screws and rods used. Pedicle screws with rods construct were also used in all of the remaining cases of posterior spinal fusion in the thoracic and lumbar spine, except in one patient who had surgery in 2007 in which a hybrid construct with Wisconsin wire in the upper thoracic spine and pedicle screws in the lumbar spine was used.

In eight patients with tumor located at the thoracic or lumbar spine who received posterior stabilization, an additional anterior support was given. Pedicle screws and rod constructs were made, spanning two vertebrae above and below the tumor. The tumor was removed posteriorly through a costotransversectomy, and a metal mesh cage was inserted posteriorly to reconstruct the vertebra.

In total, 63 patients with tumor involvement at the thoracic and lumbar spine received posterior stabilization, with eight cases having an additional anterior support. Fusion materials in the form of autologous or artificial bone substitute were used in those cases with anterior support using a metal mesh cage. The use of fusion materials in posterior-only stabilization cases was decided at the discretion of the operating surgeon. Overall, fusion materials were used in 35 patients and 53 patients underwent nonfusion instrumented surgery.

Among the 88 patients, nine patients (10.2\%) developed radiographic features of implant failure, with three patients surviving at the time of data retrieval on September 13, 2019. Among the nine cases, two cases had progressive kyphosis and three cases demonstrated cage subsidence. The remaining four cases had halos around the screws. There was no case of implant breakage. Table 6 presents the details of the nine patients who developed implant failure. Three patients required additional surgery. The first patient developed progressive kyphosis, resulting in 

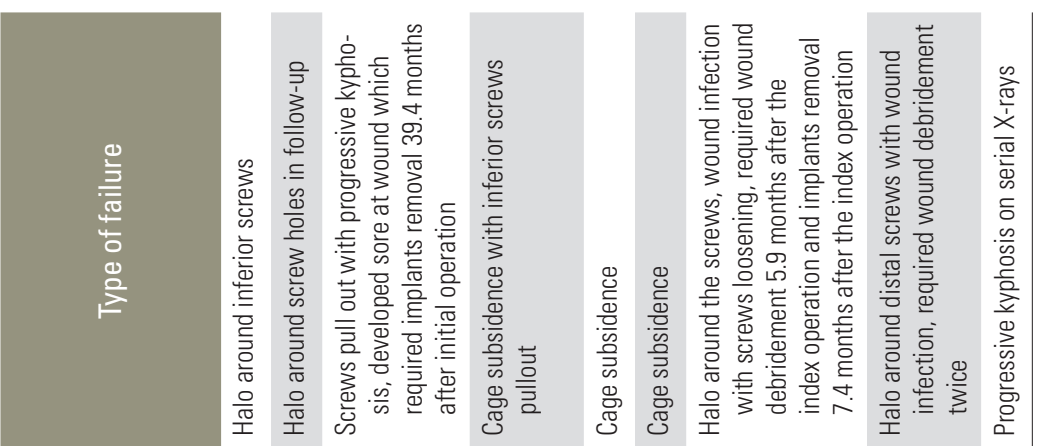

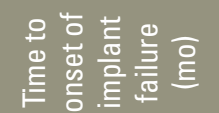

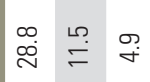

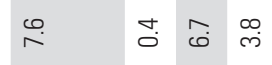

$\stackrel{+}{+}$

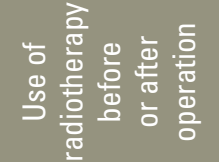

$2 \%$

$2 \quad \stackrel{0}{2}$

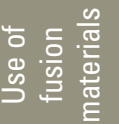

$2 \frac{2}{2}$

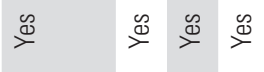

离

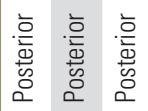

䇋它

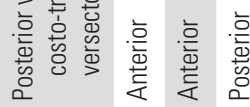

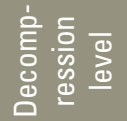

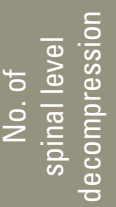

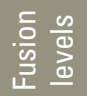

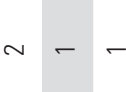

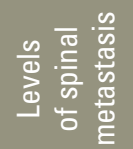

誉虽草

要是是

$=m \stackrel{\circ}{\circ}$

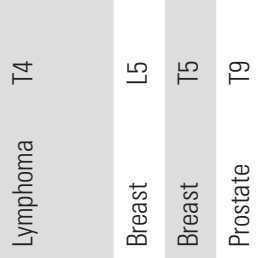

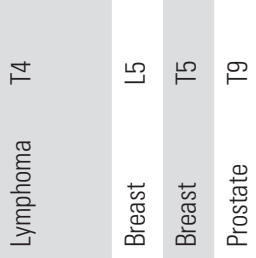

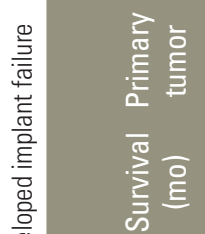

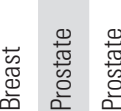

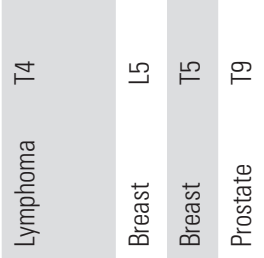

ֻ

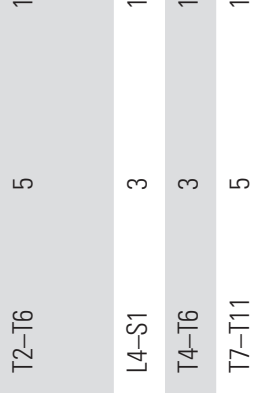

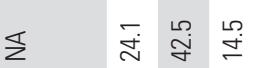

妾孚尔

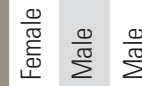

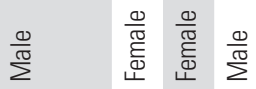

흠

$\frac{\overline{0}}{\frac{0}{4}}$

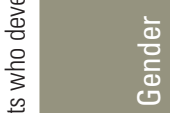

它

षํㅡㅇ

항

g

ณ

$\stackrel{\infty}{\dddot{2}} \quad \frac{0}{2}$

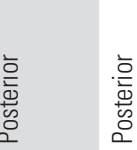

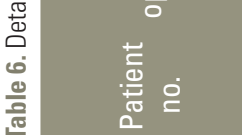
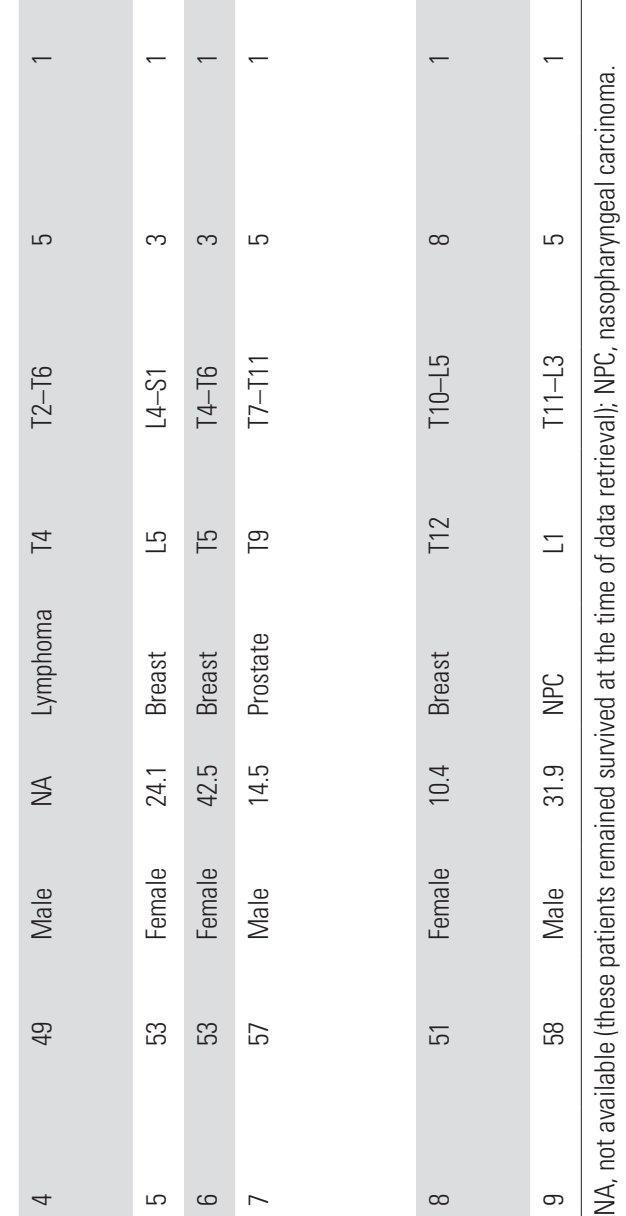
Table 7. Logistic regression analysis of potential risk factors on implant failure in spine metastasis

\begin{tabular}{|c|c|c|c|c|}
\hline Independent variable & $r^{2}$ & Odds ratio ( $95 \%$ confidence interval) & Standard error & $p$-value \\
\hline Age at operation & $<0.01$ & $0.99(0.93-1.06)$ & 0.03 & 0.99 \\
\hline Gender & $<0.01$ & $0.82(0.21-3.28)$ & 0.71 & 0.78 \\
\hline Survival period & $<0.01$ & $1.00(1.00-1.00)$ & 0.00 & 0.61 \\
\hline Primary tumor (ref: slow growth) & 0.15 & & & \\
\hline Moderate growth & & $0.00(0.00-0.00)$ & - & 1.00 \\
\hline Rapid growth & & $0.00(0.00-0.00)$ & - & 1.00 \\
\hline Spine level involved (ref: junction area) & 0.01 & & & \\
\hline Semi-rigid spine & & $0.87(0.20-3.82)$ & 0.76 & 0.86 \\
\hline Mobile spine & & $0.32(0.03-3.09)$ & 1.16 & 0.33 \\
\hline Construct length & $<0.01$ & $0.91(0.60-1.36)$ & 0.21 & 0.63 \\
\hline Levels of decompression & 0.04 & $0.22(0.03-1.77)$ & 1.07 & 0.15 \\
\hline Utilization of fusion materials (ref: no) & 0.01 & & & \\
\hline Yes & & $2.04(0.51-8.21)$ & 0.71 & 0.32 \\
\hline Application of radiotherapy either before or after spine operation (ref: no) & 0.05 & & & \\
\hline Yes & & $0.09(0.01-0.75)$ & 1.08 & 0.03 \\
\hline
\end{tabular}

Dependent variable: implant failure.

$r^{2}$, coefficient of multiple determination; Ref, reference.

a sore over the wound. Implant removal was performed 39.4 months after the initial operation. The second case developed a deep-seated wound infection, spreading to the implants and requiring wound debridement 5.9 months after the index operation and finally implant removal 7.4 months after the index operation. The third case developed a superficial wound infection and required wound debridement twice but did not require implant removal. The wound infection was superficial, and there was no evidence of the infection tracking to the implant upon wound debridement. None of the patients who developed implant failure required revision fixation. Of the six cases that did not require further operation, all were asymptomatic for implant failure.

The results of logistic regression analyses are tabulated in Table 7. All nine potential risk factors were used to look for any significant relationship. Of all the risk factors, only the use of either preoperative or postoperative radiotherapy reached a statistical significance, with implant failure being less likely to develop following preoperative or postoperative radiotherapy (odds ratio, 0.09; $p=0.03$ ). Statistical significance was not reached when considering posterior only. All nine cases that developed implant failure had a slow-growing primary tumor type. However, in analysis, the aggressiveness of the tumor or patient's survival time did not reach statistical significance.

\section{Discussion}

In our study, the overall incidence of symptomatic implant failure requiring revision surgery for implant removal was $2.3 \%$ (two out of 88 cases) with one of the cases associated with implant infection. The incidence of asymptomatic implant failure was $6.8 \%$ (six out of 88 cases). Our results suggest that the use of radiotherapy either before or after the spinal operation is associated with a reduced incidence of implant failure.

The quoted incidence of implant failure after surgery for spinal metastases is approximately $2 \%-8 \%$ [10-15]. Patients with implant failure may or may not be symptomatic, and not all patients with implant failure require further revision surgery [10]. In the review by Amankulor et al. [11] of the incidence and patterns of hardware failure after separation surgery in patients with spinal metastatic tumors, nine out of 318 patients (2.8\%) exhibited signs and symptoms of hardware failure and required revision of the instrumentation. The authors examined whether gender, construct length, presence of chest wall resection, multiple operations, kyphoplasty, and inclusion of junctional level within the construct had any relationship to the 
development of symptomatic implant failure. They found that patients with prior or concomitant chest wall resection and a construct length that spanned more than six spinal segments were more likely to experience implant failure leading to revision surgery. In another review by Drakhshandeh et al. [12], of 27 patients who underwent separation surgery with posterior spinal decompression and instrumentation without fusion, none developed implant failure in their postoperative follow-up as evidenced on CT scan; thus, no patient required revision surgery. In the review by Bellato et al. [13], of 105 patients who underwent spinal decompression and fixation for the treatment of spinal metastases using a posterior approach, without attempted arthrodesis, nine patients (8\%) developed implant failure, but none of these patients required revision surgery. In the study by Pedreira et al. [14], of 159 patients who underwent surgical resection of metastatic spine lesion, three patients (1.9\%) developed implant failure requiring revision surgery. The authors also analyzed several potential risk factors for implant failure leading to revision surgery, including the presence of bone, visceral and brain metastasis, patient's disability as measured by the modified Rankin scale, previous systemic chemotherapy or spine radiation, and the mean survival. They found that patients who had received radiation to the spine before the operation were associated with a higher risk of implant failure development leading to revision surgery. There was a trend indicating that patients with a longer life expectancy were more likely to experience hardware failure, but this was not statistically significant.

Our results appear contrary to some previous studies, in which a long construct length is not associated with an increased incidence of implant failure and that the use of radiotherapy either before or after the spinal operation is associated with a reduced incidence of implant failure. Radiotherapy is known to have a harmful effect on the bone. However, after the destruction of tumor cells by radiotherapy, recalcification of the affected vertebra occurs after 1-2 months [10]. The reconstitution of the bone stock after radiotherapy can increase the load-sharing ability of the vertebra, and this may explain our result of a reduced implant failure rate after radiotherapy.

There are some limitations in our study. First, our case number is relatively small, and for this reason, in statistical analyses, we included cases with different approaches, with or without removal and reconstruction of the vertebral body. A larger sample size with subgroup analyses of different approaches is necessary to provide a specific conclusion. Second, our cases were recruited within a 10-year interval from 2007 to 2017; advances in target therapy and radiotherapy techniques during this period have improved the survival rates of some of the tumors previously thought to be associated with poor prognosis. The improvement in survival rate may increase the implant failure rate of spinal metastasis surgery, in particular for those cases where fusion material is not used.

\section{Conclusions}

The development of radiological signs of implant failure after instrumented surgery for the treatment of spinal metastasis is common. However, symptomatic implant failure leading to revision surgery is uncommon. Our findings suggest that radiotherapy, either before or after spinal surgery, is not associated with the development of implant failure.

\section{Conflict of Interest}

No potential conflict of interest relevant to this article was reported.

\section{References}

1. Lee CS, Jung CH. Metastatic spinal tumor. Asian Spine J 2012;6:71-87.

2. Sciubba DM, Gokaslan ZL. Diagnosis and management of metastatic spine disease. Surg Oncol 2006;15:141-51.

3. Ecker RD, Endo T, Wetjen NM, Krauss WE. Diagnosis and treatment of vertebral column metastases. Mayo Clin Proc 2005;80:1177-86.

4. Tomita K, Kawahara N, Murakami H, Demura S. Total en bloc spondylectomy for spinal tumors: improvement of the technique and its associated basic background. J Orthop Sci 2006;11:3-12.

5. Lasak JM, Gorecki JP. The history of stereotactic radiosurgery and radiotherapy. Otolaryngol Clin North Am 2009;42:593-9.

6. Moussazadeh N, Laufer I, Yamada Y, Bilsky MH. Separation surgery for spinal metastases: effect of spinal radiosurgery on surgical treatment goals. Cancer Control 2014;21:168-74.

7. Raizman NM, O’Brien JR, Poehling-Monaghan KL, 
Yu WD. Pseudarthrosis of the spine. J Am Acad Orthop Surg 2009;17:494-503.

8. Choi D, Bilsky M, Fehlings M, Fisher C, Gokaslan Z. Spine oncology-metastatic spine tumors. Neurosurgery 2017;80:S131-7.

9. Vrionis FD, Small J. Surgical management of metastatic spinal neoplasms. Neurosurg Focus 2003; 15:E12.

10. Kumar N, Patel R, Wadhwa AC, et al. Basic concepts in metal work failure after metastatic spine tumour surgery. Eur Spine J 2018;27:806-14.

11. Amankulor NM, Xu R, Iorgulescu JB, et al. The incidence and patterns of hardware failure after separation surgery in patients with spinal metastatic tumors. Spine J 2014;14:1850-9.

12. Drakhshandeh D, Miller JA, Fabiano AJ. Instrumented spinal stabilization without fusion for spinal metastatic disease. World Neurosurg 2018;111:e4039.

13. Bellato RT, Teixeira WG, Torelli AG, Cristante AF, de Barros TE, de Camargo OP. Late failure of posterior fixation without bone fusion for vertebral metastases. Acta Ortop Bras 2015;23:303-6.
14. Pedreira R, Abu-Bonsrah N, Karim Ahmed A, et al. Hardware failure in patients with metastatic cancer to the spine. J Clin Neurosci 2017;45:166-71.

15. Park SJ, Lee KH, Lee CS, et al. Instrumented surgical treatment for metastatic spinal tumors: is fusion necessary? J Neurosurg Spine 2019:1-9.

16. Tomita K, Kawahara N, Kobayashi T, Yoshida A, Murakami H, Akamaru T. Surgical strategy for spinal metastases. Spine (Phila Pa 1976) 2001;26:298-306.

17. Katagiri H, Okada R, Takagi T, et al. New prognostic factors and scoring system for patients with skeletal metastasis. Cancer Med 2014;3:1359-67.

18. Yeung YN, Cheung KK, Lam TC, Cheng HO, Chow YY. A study of the predictive value of the modified Tokuhashi score in metastatic spinal tumour causing cord compression in a Southern Chinese population. J Orthop Trauma Rehabil 2014;18:15-21.

19. Fisher CG, DiPaola CP, Ryken TC, et al. A novel classification system for spinal instability in neoplastic disease: an evidence-based approach and expert consensus from the Spine Oncology Study Group. Spine (Phila Pa 1976) 2010;35:E1221-9. 